Total colonoscopy was recorded in 21 (84\%) AP and in 43 (95.6\%) NP using MEI. The median distance to the caecum in AP was $104 \mathrm{~cm}$ (IQ range, 93.7-130.5) as compared to $87 \mathrm{~cm}(79-100)$ in NP $(\mathrm{p}=0.0009$, Mann Whitney $\mathrm{U})$. The median maximum length of colonoscope inserted was $127.5 \mathrm{~cm}(110-155.1)$ in AP compared to $110 \mathrm{~cm}(105-120)$ in NP $(\mathrm{p}=0.0023)$. The greatest difference was in the median length of colonoscope inserted to reach the sigmoiddescending junction, $78.5 \mathrm{~cm}(62.9-94.1)$ in AP compared to $51.3 \mathrm{~cm}$ (42.4-61.5) in NP $(p<0.0001)$. This was associated with greater complex looping in the sigmoid colon in AP (5/24) than in NP $(1 / 44)(\mathrm{p}=0.0118$, chi square). The median time to the caecum was $772 \mathrm{sec}(613.5-1063)$ in AP compared to $563.5 \mathrm{sec}(400.8-853.6)$ in NP $(p=0.0267$, Mann Whitney U).

Conclusion: Patients with acromegaly are more difficult to colonoscope than normal patients because they have longer colons, which are more prone to loop formation.

\section{PATIENT PAIN DURING COLONOSCOPY_AN ANALYSIS USING MAGNETIC ENDOSCOPE IMAGING (MEI)}

S.G. Shah, C.J. Thapar, J.C. Brooker, C.B. Williams, B.P. Saunders. Wolfson Unit for Endoscopy, St Mark's Hospital, Watford Road, Harrow, Middlesex, UK

Background: Colonoscopy is generally perceived as being a painful procedure. Stretching of the peritoneal attachments from looping of the instrument shaft, overinsufflation, the degree of torque/force applied to the colonoscope shaft, and patient pain threshold are contributory factors.

Aims: To determine the frequency of pain episodes experienced and the corresponding colonoscope configuration during colonoscopy.

Methods: Consecutive out-patients undergoing colonoscopy were studied. Patients with previous colonic resections were excluded. Procedures were commenced with buscopan only and patient sedation/ analgesia self-administered whenever significant discomfort was experienced, using a PCA syringe pump. All 'demands' were correlated with the MEI record, which was subsequently analysed.

Results: Pain with the instrument tip in the rectum accounted for $1 \%$ of the total demands (5/675). $77 \%(521 / 675)$ of all demands occurred with the colonoscope tip in the sigmoid colon, coinciding with looping (340), stretching of the apex of the sigmoid colon (77), pulling back/shortening colon (30), straightening of a loop (27), sigmoid fixation (2), and with no visible loop (45). $7 \%$ (47/675) of demands occurred with the colonoscope tip in the descending colon, coinciding with sigmoid looping (31), straightening of a loop (10) or with a straight scope (6). $6 \%(39 / 675)$ of demands coincided with either recurrent sigmoid looping (28) or sigmoid stretch (11) at the splenic flexure. In the transverse colon $5 \%$ of the total demands (33/ 675) were secondary to either looping of the sigmoid anteriorly (12), deep transverse looping alone (7), both deep transverse \& sigmoid looping (6), straightening of a loop (2), or no visible loop (5). Demands were least frequent in the proximal colon (4\% of total (30/ 675)) and coincided with either sigmoid looping (10) or deep transverse/sigmoid looping (13) in the majority. 7 demands were associated either with straightening of a transverse loop or with no visible loop, with the colonoscope tip in the caecal pole.

Conclusions: The majority of pain/discomfort episodes (85\%) experienced were due to looping of the colonoscope shaft, most frequently in the sigmoid colon, with overinsufflation being an infrequent cause ( $9 \%$ ). Use of MEI may improve pain control by enabling the endoscopist to 'target' patient analgesia, pre-empting any pain episodes.

\section{INTRINSIC ANTI-ENDOTOXIN ANTIBODY MAY BE PROTECTIVE IN INFLAMMATORY BOWEL DISEASE PATIENTS UNDERGOING COLONOSCOPY}

P.J.D. Neilly, M. Eames, S.J. Kirk, K.R. Gardiner. Dept of Surgery, Queen's University of Belfast and Royal Victoria Hospital, Belfast, UK

Introduction: Bacteraemia and endotoxaemia may occur following lower gastrointestinal endoscopy. Endotoxaemia is a frequent feature of inflammatory bowel disease (IBD) but there is no apparent increase in morbidity following colonoscopy. This study investigates the hypothesis that anti-endotoxin antibody concentrations are elevated in IBD and may therefore protect patients undergoing colonoscopy.

Methodology: Forty-four patients requiring lower gastrointestinal endoscopy were investigated. Blood was taken before endoscopy to assess IBD severity, endotoxin and endotoxin-core antibody (Endo$\mathrm{CAb}$ ) concentrations and bacteriological status. Further samples were collected at 1 hour and 1 week after endoscopy for bacteriology and measurement of endotoxin and EndoCAb concentrations.

Results: Seven patients with disease other than colitis were excluded from study. Comparing normal and IBD patients before endoscopy there was no significant difference in the incidence of bacteraemia or endotoxaemia. Endotoxin concentrations were increased in controls 1 hour after endoscopy $(p=0.02)$ but endotoxin concentrations were not significantly higher in colitics. The colitics however had a higher incidence of bacteraemia 1 hour after endoscopy $(p=0.05)$, being most evident in those with severe disease $(p=0.01)$. Compared with controls IBD patients had higher EndoCAb concentrations before, 1 hour and 1 week after endoscopy $(p<0.02)$. Patients with more severe disease also had higher EndoCAb concentrations than those with mild disease 1 hour after endoscopy ( $p=0.03$ ).

Conclusion: Antibodies to endotoxin are higher in IBD patients and systemic concentrations correlate with disease activity. No significant change in endotoxin concentrations in these patients following endoscopy, despite an increased incidence of bacteraemia, would suggest that anti-endotoxin antibodies have a protective effect.

\section{Biliary Posters: 378-388}

\section{ENDOSCOPIC SURGERY FOR CHOLEDOCHOLITHIASIS WITHOUT SUBSEQUENT CHOLECYSTECTOMY- ANALYSIS OF LONG-TERM RESULTS}

K. Nigam, R.H. Kennedy. Dept of Surgery, Yeovil District Hospital, Yeovil, UK

Aims: To analyse the results of endoscopic treatment of bile duct (BD) stones without subsequent cholecystectomy in patients over 64 years old or those unfit for surgery.

Methods: All consecutive patients undergoing endoscopic bile duct clearance during six years to 31.12 .98 were analysed from various prospectively collected data sources. Statistical analysis-chisquared test with Yate's correction.

Results: 174 patients were included (median age of 76 years) of whom 13 were $<65$ years but were unfit for surgery (age range 49-64). Successful endoscopic sphincterotomy (ES) was achieved in 93\% of patients but 29 required surgery to clear the bile duct. Following endoscopic duct clearance, 143 patients were discharged with gallbladders in situ. Mean follow-up was 41.2 months. $15 \%$ required subsequent cholecystectomty (due to acute cholecystitis in 8 and recurrent biliary colic in 8 patients). 13\% required further endoscopic BD surgery. There was no recurrence of biliary pancreatitis. $58 \%$ of patients in the age group 65-70 yrs at initial presentation required subsequent intervention (SI) as compared to $19.5 \%$ in patients over 70 ( $\mathrm{p}$ value $=$ 0.0003). Overall complication rate of ERCP/ES was $5 \%$.

Conclusions: ERCP/ES alone without subsequent cholecystectomy is safe and effective for treating high-risk patients with choledocholithiasis, effectively prevents recurrence of biliary pancreatitis and does not increase the risk of subsequent acute cholecystitis. Subsequent cholecystectomy rate of $15 \%$ is higher than $<11 \%$ previously reported. More than $50 \%$ patients in $65-70$ yrs age group will need further treatment after endoscopic BD clearance. Majority of SI take will place in first 2 years following endoscopic BD clearance. Rate of SI is not related to the length of follow-up. Comparison with laparoscopic bile duct surgery is needed.

\section{GALLBLADDER ENLARGEMENT AFTER MAJOR SURGERY}

P.V. Gallagher ${ }^{1}$, S.T. Elliott ${ }^{2}$, R.M. Charnley ${ }^{1}$. Depts of Surgery ${ }^{1}$ and Radiology ${ }^{2}$, Freeman Hospital, Newcastle upon Tyne NE19 2HP, UK

Background: Altered gallbladder motility has been implicated in the pathogenesis of gallstones in conditions such as pregnancy or during parenteral nutrition. An increased incidence of gallstones occurs after certain major surgical operations. Alterations in gallbladder motility have not previously been studied in the peri-operative period.

Aim: To measure gallbladder motility in subjects undergoing major non- gastrointestinal surgery in the peri-operative period.

Methods: Subjects undergoing aortic surgery $(n=12)$ and cardiac surgery $(n=19)$ had fasting gallbladder volumes measured immediately before surgery and again after being established on a normal diet 
before discharge from hospital. Ultrasound was used to make triplicate measures of gallbladder dimensions. The mean of each dimension was used to calculate gallbladder volume by the ellipsoid method.

Results: Mean fasting gallbladder volumes had increased by $270 \%$ in the aortic group and $290 \%$ in the cardiac group after surgery, despite the subjects taking diet (see table 1).

Abstract 379, Table 1

\begin{tabular}{lll}
\hline & Aortic & Cardiac \\
\hline $\begin{array}{l}\text { Pre-op mean volume (standard deviation) } \\
\begin{array}{l}\text { Median number of days USS performed after } \\
\text { diet commenced (range) }\end{array}\end{array}$ & $23.3 \mathrm{ml}(8.0)$ & $20.7 \mathrm{ml}(11.5)$ \\
$\begin{array}{l}\text { Median number of days after surgery } \\
\text { ultrasound performed (range) }\end{array}$ & $3.0(1-16)$ & $3.3(1-6)$ \\
$\begin{array}{l}\text { Post-op mean volume (standard deviation) } \\
\begin{array}{l}\text { Paired t-test (pre-op volume vs. post-op } \\
\text { volumes) }\end{array}\end{array}$ & $63.0(5-26)$ & $5.8(4-8)$ \\
& $\mathrm{Pl}<0.001$ & $\mathrm{P}<0.001$ \\
\hline
\end{tabular}

Conclusions: Despite taking diet, fasting gallbladder volumes are increased in patients undergoing major non-gastrointestinal surgery until at least the time of discharge from hospital. Altered gallbladder motility following surgery may produce biliary stasis contributing to an increased incidence of gallstones in these patients.

\section{PREDICTING COMMON BILE DUCT STONES BEFORE PREOPERATIVE ERCP}

A. Li, P.G. Wheeler. Dept of Gastroenterology, William Harvey Hospital, Ashford, Kent, TN24 OLZ, UK

Background: A common indication for ERCP is to determine the presence of common bile duct (CBD) stones prior to laparoscopic cholecystectomy. Previous attempts have been made to avoid unnecessary ERCP using preoperative clinical and investigative parameters to select out patients with low probability of CBD stones. A retrospective audit was performed in a district hospital setting and selection criteria were applied to the data.

Methods: All patients undergoing ERCP prior to laparoscopic cholecystectomy over a 3-year period were identified. Exclusion criteria were ERCP diagnoses of biliary stricture or hepatobiliary neoplasm. Only those with ultrasound reports and liver biochemistry results within 1 month prior to ERCP were included. 108 patients were included. Retrospectively, selection criteria were applied suggesting high probability of CBD stone prior to ERCP. These were: persistent as opposed to resolving jaundice within 1 month prior to ERCP, dilated CBD $>7 \mathrm{~mm}$ diameter, or CBD stone on ultrasound, history of pancreatitis or cholangitis.

Results: 63 out of $108(58 \%)$ patients with CBD stones queried prior to laparoscopic cholecystectomy proved to have them on ERCP. Using retrospective selection criteria, 53 of these $63(84 \%)$ patients with CBD stone were correctly identified as requiring preoperative ERCP. 31 out of the remaining 45 patients who did not have CBD stone but who were predicted to have them would have undergone unnecessary ERCP. Prediction of CBD stones thus has sensitivity $(84 \%)$ but not specificity (31\%). Individual predictors of CBD stones were: history of cholangitis, persistent jaundice and CBD dilatation or CBD stone on ultrasound. Age, sex, and history of pancreatitis were not predictive of CBD stones.

Conclusions: It is possible, using basic clinical parameters to predict the presence of CBD stone prior to laparoscopic cholecystectomy and reduce the numbers of preoperative ERCP.

\section{MAGNETIC RESONANCE CHOLANGIOPANCREATOGRAPHY VERSUS ENDOSCOPIC RETROGRADE CHOLANGIOPANCREATOGRAPHY IN THE DIAGNOSIS OF CHOLEDOCHOLITHIASIS}

N. Griffin ${ }^{1}$, M. Wastie ${ }^{2}$, K. Dunn ${ }^{2}$, S.D. Ryder ${ }^{3}$, I.J. Beckingham ${ }^{1}$. Depts of Surgery ${ }^{1}$, Medicine $^{3}$ and Radiology ${ }^{2}$, Queen's Medical Centre, University Hospital, Nottingham, UK

Background: Over the last few decades, endoscopic retrograde cholangiopancreatography (ERCP) has become established as the gold standard in imaging of the biliary tree. More recently magnetic resonance cholangiopancreatography (MRCP) has been introduced as a new, non-invasive imaging modality for the detection of common bile duct stones and other pathology related to the biliary tract and pancreas.

Methods: A prospective study was carried out of 133 patients referred for both investigations to compare the results of ERCP and MRCP in determining the presence of common bile duct stones prior to laparoscopic cholecystectomy.

Results: 18 patients were excluded from the analysis as ERCP was unsuccessful in 8 patients and MRCP was not possible in a further 10 patients. There were 6 false negative results with MRCP and in 5 of these the calculi were less than $5 \mathrm{~mm}$ in diameter. MRCP showed a sensitivity of $84 \%$, specificity of $96 \%$, positive predictive value of $91 \%$ and negative predictive value of $93 \%$ when compared to ERCP as the gold standard.

Conclusion: MRCP has both high sensitivity and specificity for stones greater than $5 \mathrm{~mm}$ in diameter and should be the first line investigation in patients with gallstones and abnormal liver function tests. Adoption of this guideline at our institution would result in a $9 \%$ reduction in the number of ERCP's performed.

\section{RAPID ASSESSMENT OF THE JAUNDICED PATIENT: THE JAUNDICE HOTLINE}

J. Mitchell ${ }^{1}$, S.H. Hussaini ${ }^{1}$, D. McGovern ${ }^{2}$, G. Masketll', R. Farrow ${ }^{1}$, H.R. Dalton ${ }^{1} .{ }^{1}$ Gastroenterology Unit, Royal Cornwall Hospital, Treliske, Truro; ${ }^{2}$ fohn Radcliffe Hospital, Oxford, UK

Introduction: In order to meet the challenge of increasing workloads and the governments "two week" rule, innovative reorganisation of existing health services may be required. These factors and a desire to improve our referral system for the acutely jaundiced patient, led to the establishment of an open access jaundice clinic; the Jaundice Hotline.

Methods: Referrals are made via a dedicated 24 hour phone line. Patients are assigned to the next available twice weekly clinic. Following a full history and examination, an ultrasound examination is performed by a consultant gastrointestinal radiologist. Appropriate blood tests are taken and the patient is then assigned to the next ERCP list, early outpatient review or direct admission.

Results: In the first year 107 patients were seen. 62 patients (58\%) had biliary obstruction. The mean time between referral and consultation was 2.5 days. Patients who required an ERCP waited a mean time of 5.7 days. In the 69 patients who required hospital admission, the mean hospital stay was 6.1 days. The majority of these stayed 1 or two days for ERCP. This compares favourably with audit data from 1996 which showed a mean hospital stay of 11.5 days. $97 \%$ of patients and $95 \%$ of primary care practices rated the service as above average or excellent.

Conclusions: This approach to the jaundiced patient results in rapid assessment, diagnosis and treatment as well as reducing hospital stay. Novel reorganisation of existing health services at minimal extra cost will be important to high quality health service provision in the face of the UK governments "two week" cancer ruling.

\section{THE FREQUENCY OF SPONTANEOUS PASSAGE OF BILE DUCT STONES AND RELATION TO CLINICAL PRESENTATION}

S.E. Tranter, M.H. Thompson. Dept of Surgery, Southmead Hospital, Bristol, $U K$

Aims: Little is known about the spontaneous passage of bile duct stones. The aim of this study is to determine the rate of spontaneous stone passage and relate it to the clinical presentation of the bile duct stone.

Methods: Prospectively collected data was studied on a total of 1051 consecutive patients undergoing laparoscopic cholecystectomy with or without laparoscopic common duct exploration (LCDE). Comparisons were made between 142 patients with common bile duct stones (CBDS); 519 patients who had no previous or current evidence of duct stones and 390 patients who had good evidence of previous duct stones but not present at the time of cholecystectomy. The evidence used for previous duct stones included a good history of jaundice, a raised serum amylase, abnormal pre-operative liver function tests and/or a dilated common bile duct. We have assumed that this group underwent spontaneous passage of bile duct stones. 
Results: $51 \%$ of patients undergoing laparoscopic cholecystectomy had a history of previous or current CBDS: $73 \%$ of these passed their ductal stones spontaneously prior to operation. Patients presenting with pancreatitis had a statistically significant $80 \%$ chance of passing their stones spontaneously $(p<0.001)$. Those presenting with jaundice had only a $55 \%$ chance of spontaneous passage; the remainder in each group required LCDE or endoscopic sphincterotomy. All patients with cholangitis had CBDS at the time of operation.

Conclusions: The majority of patients (almost 3 in 4) with CBDS passed their stones spontaneously. Four out of five patients with pancreatitis passed their stones spontaneously, in contrast to patients with jaundice who were less likely to undergo spontaneous resolution. Cholangitis always implied the presence of duct stones.

\section{ASSOCIATION OF PERIAMPULLARY DUODENAL DIVERTICULAE WITH BILE DUCT STONES AND TECHNICAL SUCCESS AT ERCP}

M. Kelly, T.C.K. Tham. Division of Gastroenterology, Ulster Hospital Dundonald, Belfast, N. Ireland, UK

Background and aim: Periampullary diverticulae are thought to be associated with bile duct stones (BDS). However studies to date have been inconclusive as they do not take into account the influence of age. Our study analysed the association of diverticulae with BDS and technical success of ERCP.

Methods: Patients were prospectively entered into a database. 415 underwent ERCPs. 83 (20\%) of these patients had diverticulae. The aged matched control group had 261 patients. The Chi square test was used to analyse the results.

Results: See table 1.

Abstract 384, Table 1

\begin{tabular}{llll}
\hline & Diverticulae & Controls & P value \\
\hline Number & 83 & 261 & \\
Age & $73(26-89)$ & $72(51-93)$ & N.S. \\
Sex & 34 male, 49 fem & $106 \mathrm{M}, 155 \mathrm{~F}$ & \\
Total with BDS & $53(64 \%)$ & $86(33 \%)$ & $\mathrm{P}<.0001$ \\
BDS + gallstones & 44 & 74 & $\mathrm{P}=0.003$ \\
Primary BDS & 7 & 2 & $\mathrm{P}=0.005$ \\
BDS, previous cholecystectomy & 2 & 10 & $\mathrm{P}=0.19$ \\
Gallstones/cholecystectomies & $56(67 \%)$ & $157(60 \%)$ & $\mathrm{P}=0.29$ \\
Biliary strictures & $7(8 \%)$ & $45(17 \%)$ & $\mathrm{P}=0.07$ \\
H/O pancreatitis & $8(10 \%)$ & $28(11 \%)$ & $\mathrm{P}=0.94$ \\
Cannulation & $78 / 83(94 \%)$ & $245 / 261(94 \%)$ & $\mathrm{P}=0.82$ \\
Sphincterotomies & $49 / 51(96 \%)$ & $85 / 87(98 \%)$ & $\mathrm{P}=0.98$ \\
Stone extraction & $35 / 37(94 \%)$ & $61 / 69(88 \%)$ & $\mathrm{P}=0.49$ \\
\hline
\end{tabular}

Conclusions: Duodenal diverticulae are associated with an increased incidence of both primary and secondary bile duct stones. Duodenal diverticulae are not associated with pancreatitis. Duodenal diverticulae did not cause any technical difficulties at ERCP.

\section{SPHINCTER OF ODDI MANOMETRY-USE AND SAFETY}

W. Jackson, K.R. Wedgwood. Dept of Upper GI Physiology, Castle Hill Hospital, Cottingham, Hull, UK

Introduction: The sphincter of oddi ( $\mathrm{SO}$ ) regulates the flow of pancreatic and bile juice into the duodenum and prevents duodenal reflux. Sphincter of oddi manometry (SOM) can be used to diagnose dysfunction of the SO and the bilary tract. Traditionally water perfused catheters (WPC) are used for SOM which is performed when the patient routinely under goes ERCP but can result in acute pancreatitis (AP).

Aim: To assess a solid state pressure transducer catheter (SSPTC) in the bilary tract, monitoring patients' status of pancreatitis pre and post SOM using a SSPTC, and to determine whether patients (Pts) symptoms can be attributable to abnormal SOM and be relieved by sphincterotomy.

Method: Symptomatic Pts referred for ERCP also underwent SOM using a Gaeltec SSPTC. Duodenal and SO basal pressure, SO wave amplitude (WA) and wave frequency (WF) were recorded. Those Pts in whom SOM was deemed as abnormal e.g. hypertensive basal or peak pressure or abnormal WA or WF underwent sphincterotomy.
Results: 32 Pts (20M, 12F-age range 19-91) entered the study. Mean duodenal pressure was $6 \mathrm{mmHg}$ (range 0-13). In $12 \mathrm{Pts}$ SO function exhibited normal characteristics with mean SO basal pressure of $19 \mathrm{mmHg}$ (range 8-32), WA $128 \mathrm{mmHg}$ (range 99-163) and WF of $4 / \mathrm{min}$ (range 2-6). 20 Pts had abnormal characteristics. Combinations of 1 or more abnormalities were seen, high SO basal pressures in 5 Pts of $58 \mathrm{mmHg}$ (range 42-89). High WA in 3 Pts of $245 \mathrm{mmHg}$ (range 199- >224). Low WA in $10 \mathrm{Pts}$ of $72 \mathrm{mmHg}$ (range 42-93) and abnormal WF in $12 \mathrm{Pts}$ of $12 / \mathrm{min}$ (range $7-23 / \mathrm{min}$ ). Following sphincterotomy $82 \%$ of the Pts symptoms improved while $18 \%$ remained the same. No patient developed AP with a SSPTC.

Conclusion: The use of SSPTC for SOM reduces the inherent risks of AP caused by WPC. SOM may also identify Pts with abnormal motility resulting in symptoms who may benefit from sphincterotomy.

\section{HIGH SPHINCTER PRESSURES ARE ASSOCIATED WITH LOWER ANXIETY SCORES IN SUSPECTED SPHINCTER OF ODDI DYSFUNCTION}

M. Guinane, S. Lachmanan, E. Stoner, E. Yazaki, D. Evans, C. Ainley. The Wingate Institute, London, UK

Background: The confirmatory test for sphincter of Oddi dysfunction (SOD) is manometry (SOM) which may be hazardous. When SOM is equivocal, the decision to treat by sphincterotomy, entailing further risk, is difficult to justify. The symptoms of SOD may be nonspecific and only appropriate pain is necessary for consideration of the diagnosis. SOD may coexist with functional GI disturbances, which may cause similar symptoms. Such disturbances are associated with higher anxiety and depression scores than controls.

Aims: To examine the anxiety and depression scores of patients attending for SOM compared with results of SOM.

Methods: 56 patients attending for SOM completed Hospital Anxiety and Depression questionnaires. Scores for those with unequivocal sphincter hypertension were compared with those with normal SOM findings (Mann-Whitney U).

Results: Anxiety and depression scores are shown as median (interquartile range)

Abstract 386, Table 1

\begin{tabular}{llll}
\hline & $S O D(n=22)$ & Normal $(n=34)$ & $p$ \\
\hline Anxiety & $5(4-8)$ & $8(5-11)$ & 0.04 \\
Depression & $2(2-6)$ & $4(1-7)$ & 0.43
\end{tabular}

Conclusion: Anxiety (but not depression) scores are significantly lower in those with definite SOD. The risks of investigating and treating SOD are such that alternative methods to predict the likely outcome of SOM and sphincterotomy are required. In combination with other techniques, psychological features may help indicate those patients for whom these procedures would be most beneficial.

\section{INTERPRETATION OF ERCP SPOT FILMS BY RADIOLOGISTS-IS IT NECESSARY?}

S.Z. Abbas, J.R. Lowes, D.K. George, ' J.L. Isaacs, R.H. Teague. Gastroenterology Dept, Torbay Hospital, Torquay, UK; ${ }^{1}$ Radiology Dept, Torbay Hospital, Torquay, UK

Background: It is a common practice in Britain for ERCP films to be reported by the endoscopist carrying out the procedure and also be interpreted by a radiologist afterwards. Its clinical value (and cost-effectiveness) has recently been challenged by an American tertiary centre (DDW $2000^{1}$ ).

Aim: To determine the clinical impact of radiologist's postprocedure ERCP spot films interpretation at a DGH in Britain.

Methods: 140 consecutive ERCPs performed on 115 patients by 2 endoscopists and subsequently interpreted by a single radiologist in our unit were retrospectively analysed. Their reports were compared to the ones of the radiologist's (who had the endoscopists' report available at the time of reporting) and were divided into the following 3 categories: I) complete agreement, II) clinically insignificant findings reported by the radiologist but missed by the endoscopists, and III) clinically significant findings (that may have changed the patients' management) reported by the radiologist but missed by the endoscopists. A third gastroenterologist reviewed all the reports in the categories II and III. 
Results: The mean age of the patients ( 65 males; 75 females) was 66 years (range $=27-96$ ). The rate of cannulation of the duct of interest was 127 out of 140 cases (91\%), and both endoscopists and radiologist's reports were available in 118 of these.

Abstract 387, Table 1

\begin{tabular}{llll}
\hline Categories $n(\%)$ & & & \\
\cline { 1 - 3 } & II & Total $(n)$ \\
\hline 104 & 11 & 3 & 118 \\
\hline
\end{tabular}

Conclusion: The role of radiologists' interpretation of postprocedure ERCP spot films is small but significant. We believe this procedure should continue but be subject to further review.

1. Shah RJ, Sweeney JT, Ulrich CD, Martin SP, Somogyi L. ERCP interpretation by radiologists: does it have a role? Gastrointestinal Endoscopy 2000;51(4):AB76.

\section{THE ROLE OF SPIRAL CT CHOLANGIOGRAPHY IN PATIENTS WITH ABDOMINAL PAIN POST CHOLECYSTECTOMY}

A.C. Ashdown, R. Preston, I. Morrison, A.F. Muller. Kent and Canterbury Hospital, Ethelbert Road, Canterbury, Kent CT1 3NG, UK

Introduction: Patients (pts) with biliary type abdominal pain post cholecystectomy are often referred to gastroenterologists for ERCP. In view of the recognised risks associated with this procedure, the role of spiral CT cholangiography (sCTC), which has been shown to be highly sensitive for the detection of bile duct filling defects, in addition to ultrasound (US) and liver function tests (lfts), was examined to determine if all requests were appropriate.

Methods: 20 pts ( 7 male, 13 female, age range $32-83$ yrs), in whom ERCP was not thought to be immediately indicated, were referred to sCTC. All had had a cholecystectomy within the last 5 years. No pt was jaundiced. After lfts and US had been performed, all patients had sCTC using i.v. biloscopin, reported by one consultant radiologist. Only pts shown to have biliary stones by sCTC underwent ERCP.

Results: sCTC was successful in $18 / 20$ pts (contract may not be excreted if there is abnormal liver function). 5 pts had biliary stones detected by sCTC ( 4 with normal calibre bile ducts on US); these were all confirmed at ERCP. 13 pts had a normal sCTC. Only 11 of these pts had had a previous US, each of which was normal. sCTC was normal in the 9 pts with normal lfts. 5 patients with abnormal lfts had a normal sCTC.

Conclusion: sCTC provides high resolution images of the biliary tree in the majority of patients. It is capable of picking up stones even in the presence of a non dilated biliary tree on US. sCTC should be considered in selected patients prior to ERCP and may prevent unnecessary procedures.

\section{Liver Posters: $389-410$}

\section{ORTHOTOPIC LIVER TRANSPLANTATION, PSC, IBD AND COLORECTAL CARCINOMA}

A.J. Hughes, B. Gunson, M.J. Langman, J. Neuberger.

Aim: We reviewed our centre's experience of prevalence of IBD in patients undergoing transplantation for PSC, indication for colectomy and incidence of colorectal carcinoma.

Methods: Patients were identified from a prospectively maintained database then retrospective case note analysis was undertaken.

Results: 936 patients underwent orthotopic liver transplantation between January 1982 \& February 1999 and subsequently followed up for $>12$ months. 105 were transplanted for PSC (with no evidence of cholangiocarcinoma), 78 men and 27 women with a mean age of 45.5 years were followed up for a median of 61 months (range 13-143). Pre-transplant 66 patients $(62.9 \%)$ were diagnosed with UC and $4(3.8 \%)$ with Crohns . Post-transplant this rose to $78(74.3 \%)$ with U.C. and $5(4.8 \%)$ with Crohns. Colectomy for non malignant indications: pre-transplant - 9 (2 dysplasia, 7 symptomatic), peri-operatively - 1 for dysplasia , post-operatively - 5 (1 dysplasia, 4 symptomatic). 7 patients, all with UC, developed colorectal adenocarcinoma and 1 caecal lymphoma. Incidence of colorectal adenocarcinoma in patients transplanted for PSC with an intact colon was $7.4 \%$ compared with $0.8 \%$ for the rest of transplant group $(\mathrm{p}<0.05)$ and $12.3 \%$ in patients with UC diagnosed prior to transplantation for PSC. Malignancy arose after a median of $4.5 \mathrm{yrs}$ and all had colitis for $>18$ yrs.

Conclusion: Patients with PSC and longstanding UC have a considerably increased risk of developing colorectal adenocarcinoma post liver transplantation and should be screened aggressively.

\section{WORLDWIDE TRENDS IN MORTALITY RATES FOR HEPATOBILIARY AND PANCREATIC TUMOURS, 1979-1997}

S.A. Khan ${ }^{1}$, S.D. Taylor-Robinson ${ }^{1}$, M.B. Toledano ${ }^{2}$, P. Elliott ${ }^{2}$, H.C. Thomas ${ }^{1}$. The Liver Unit, ${ }^{1}$ Dept of Medicine $A$ and ${ }^{2}$ Dept of Epidemiology, Imperial College School of Medicine, St Mary's Hospital Campus, South Wharf St, London W2 $1 P G$, UK

Background: The age-standardised mortality rate (ASMR) per 100,000 population for hepatocellular carcinoma (International Classification of Disease-9 155.0) is increasing in several countries including USA and Japan; but in England \& Wales we previously reported an increase in ASMR for intrahepatic cholangiocarcinoma (ICD-9 155.1) only. To investigate this further we analysed mortality statistics from North America, Japan, Australia and Europe to compare ASMR of subcategories of hepatobiliary and pancreatic tumours.

Methods: ASMR for men and women for subcategories of liver tumours (ICD-9 155), tumours of the gall bladder and extrahepatic biliary tree (ICD-9 156) and pancreas (ICD-9 157) from 1979 to 1997 were obtained from the World Health Organisation mortality database.

Results: We confirmed increases in ASMR for hepatocellular carcinoma in those countries were previously reported, but also found significant rises in other countries, particularly France (2.4 to 7.6). There was also an increase in ASMR for intrahepatic cholangiocarcinoma in nearly all countries studied, with large rises seen in Scotland (0.2 to 1.0$)$, Australia ( 0.1 to 0.7 ) and USA (0.2 to 0.6$)$. There was a general downward trend in extrahepatic and pancreatic malignancies, with the exception of Southern European countries and Japan.

Conclusion: We confirm previous studies of rising death rates from hepatocellular in several countries, although the trends were not uniform amongst all countries. We also present a hitherto unreported rise in mortality rates for intrahepatic cholangiocarcinoma across 4 continents. This cannot readily be explained by diagnostic transfer from other tumour types, or by improved diagnosis and reporting. Future research on cholangiocarcinoma should include epidemiological studies to examine possible case-clustering and investigation of potential aetiological agents including environmental toxins. ASMR from hepatobiliary and pancreatic tumours are not static and require close future monitoring.

\section{POSITIVE CRITERIA FOR PREDICTING MALIGNANCY IN BILIARY BRUSHING CYTOLOGY}

C. Wight, A.M. Zaitoun, I. Beckingham, J.R. Boulton-Jones, C. Dunkley, S.D. Ryder. Queen's Medical Centre, University Hospital NHS Trust, Nottingham NG7 2UH, UK

A total of 125 patients with biliary stricture and tumour masses had brushing cytology over a period of 3 years (1997-2000). One hundred and thirty five biliary brushing specimens with a known outcome were reviewed. On review an additional category of atypical probably benign was added (table). The review was carried out blind with no clinical or outcome information by two pathologists. On review the following criteria was considered indicative of malignancy: increased nuclear size, convoluted nuclear outline, abnormal chromatin pattern, increased nucleolar area and numbers, loss of cohesiveness and overlapping of nuclei. Only the negative for malignancy and malignant categories of test result were used to determine the value of the test. The results were as follows: 